\title{
Erratum: Anisotropy in the equation of state of magnetized quark matter [Phys. Rev. C 91, 065205 (2015)]
}

\author{
Débora P. Menezes, Marcus B. Pinto, and Constança Providência
}

(Received 17 October 2017; published 23 February 2018)

DOI: 10.1103/PhysRevC.97.029904

In this Erratum we present the correct result for the magnetization of the three flavor Nambu-Jona-Lasinio model which was obtained by us in the original paper. Unfortunately, the derivatives of the condensates with respect to the magnetic-field $\phi_{f}^{\prime}$, which do not contribute due the extremization conditions $\left(\partial \Omega / \partial \phi_{f}=0\right)$, were mistakenly considered in Eq. (3.4) therein. Once this mistake is corrected the relation for the magnetization reads

$$
\mathcal{M}=\sum_{f}\left(\theta_{f}^{\prime \text { mag }}+\theta_{f}^{\prime \text { med }}\right)
$$
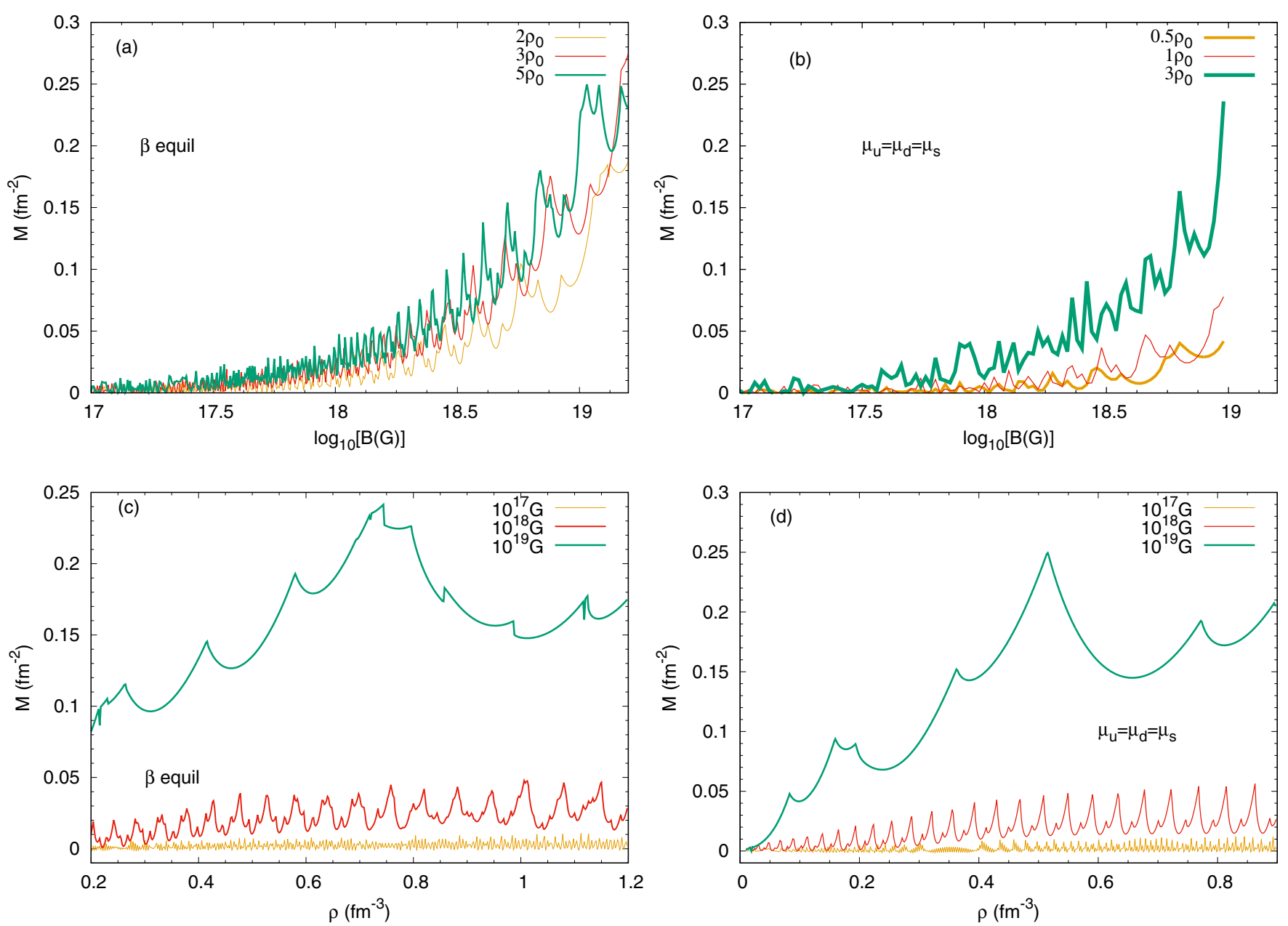

FIG. 1. Magnetization for $\beta$-equilibrium matter [panels (a) and (c)] and isochemical potential quark matter [panels (b) and (d)]. Densities of interest for each scenario are considered. In (a) and (b) the magnetization is shown as a function of $B$ and in (c) and (d) as a function of the baryonic density. 

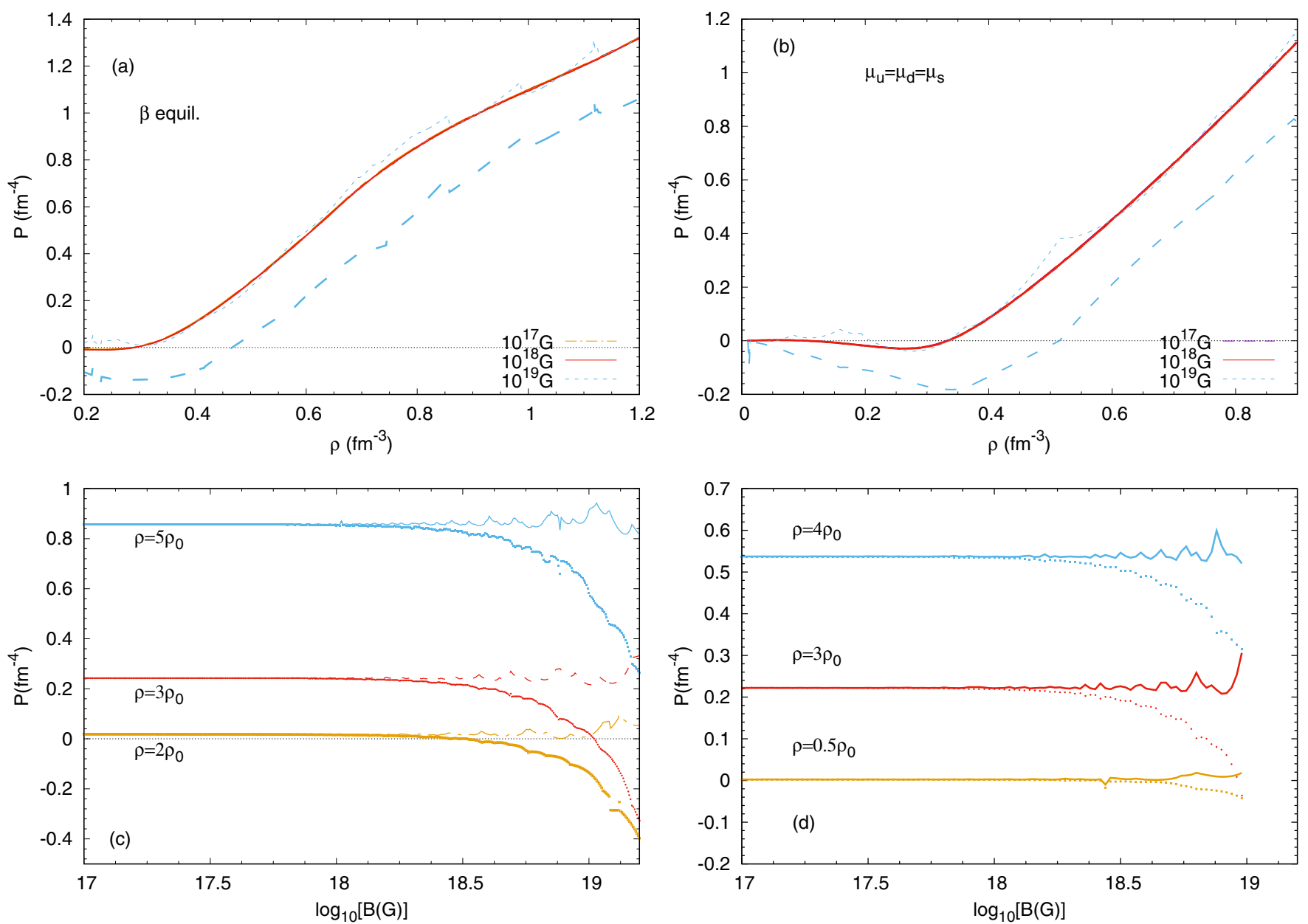

FIG. 2. Parallel (the continuous lines) and perpendicular (the marks) pressures for $\beta$-equilibrium matter [panels (a) and (c)] and isochemical potential quark matter [panels (b) and (d)]. Densities of interest for each scenario are considered. In (a) and (b) the pressures are shown as a function of the baryonic density and in (c) and (d) as a function of $B$.

where $\theta_{f}^{\prime \text { mag }}$ and $\theta_{f}^{\prime \text { med }}$ are presented in Eqs. (A2) and (A4), respectively, of the Appendix in the original paper. Therefore, only the kinetic term of the thermodynamical potential contributes to the magnetization. This expression is consistent with the one previously obtained in Ref. [1]. Using Eq. (1) we next reobtain the magnetization as well as the pressure which are shown in Figs. 1 and 2 respectively, for the same cases presented in Figs. 2 and 3 of the original paper and draw some conclusions.

We first comment on Fig. 1. Contrary to our previous findings, once the correct expression for the magnetization Eq. (1) is considered, one observes that the magnetization presents much smaller oscillations than the ones found in the original paper. Besides, the average magnetization increases with the increase in the magnetic field. The results are now very similar to the ones obtained in Ref. [2] for strange quark matter and in Ref. [3] for a gas of protons when the anomalous magnetic moment is not included. The magnetization is essentially positive for fields $B>5 \times 10^{17} \mathrm{G}$, but below that field it may get negative values in some restricted density regions (never lower than $-0.002 \mathrm{fm}^{-2}$ ). It remains possible to identify the superposition of the contributions from the different quarks, which due to their differences in mass and charge, give rise to the irregular oscillations of the magnetization. However, the onset of the strange quark does not produce the strong effects previously calculated, which was due to the derivative of the condensate, in particular, Eq. (A7) of the original paper.

The new results presented in Fig. 2 are also very much in line with the findings of Refs. [2,4,5], however different from the ones of Ref. [1]. For fields of $B \leqslant 10^{18} \mathrm{G}$ the perpendicular pressure falls on top of the parallel contribution. $P_{\perp}$ differs from $P_{\|}$ only above $B>10^{18} \mathrm{G}$, and the discontinuities present in the previous version are no longer present. As a concluding remark, we say that the anisotropic effects on the kinetic pressure are only noticeable for magnetic fields larger than $4 \times 10^{18} \mathrm{G}$ as already pointed out in Ref. [5]. Note that the anisotropies discussed in the present Erratum and associated with the quantities denominated as parallel and perpendicular pressures correspond to the space elements of the energy-momentum tensor diagonal. On the other hand, the matter pressure, a thermodynamic quantity obtained from the derivative of the partition function, is a scalar, and its value is independent of the direction considered, see, for instance, Refs. [6-8] for discussions. 
D.P.M. and M.B.P. were partially supported by Conselho Nacional de Desenvolvimento Científico e Tecnológico (CNPq-Brazil) and by Project INCT-FNA Project No. 464898/2014-5. C.P. acknowledges financial support by Fundação para a Ciência e Tecnologia (FCT-Portugal) under Project No. UID/FIS/04564/2016 and the COST action Grant No. MP1304 "NewComsptar."

[1] S. S. Avancini, V. Dexheimer, R. L. S. Farias, and V. S. Timóteo, arXiv:1709.02774.

[2] X.-G. Huang, M. Huang, D. H. Rischke, and A. Sedrakian, Phys. Rev. D 81, 045015 (2010).

[3] M. Strickland, V. Dexheimer, and D. P. Menezes, Phys. Rev. D 86, 125032 (2012).

[4] A. Rabhi, C. Providência, and J. Da Providência, Phys. Rev. C 80, 025806 (2009).
[5] V. Dexheimer, D. P. Menezes, and M. Strickland, J. Phys. G 41, 015203 (2014).

[6] D. Chatterjee, T. Elghozi, J. Novak, and M. Oertel, Mon. Not. R. Astron. Soc. 447, 3785 (2015).

[7] A. Y. Potekhin and D. G. Yakovlev, Phys. Rev. C 85, 039801 (2012).

[8] R. D. Blandford and L. Hernquist, J. Phys. C 15, 6233 (1982). 